\title{
BOUNDED WEAK SOLUTIONS OF AN ELLIPTIC-PARABOLIC NEUMANN PROBLEM
}

\author{
J. HULSHOF
}

\begin{abstract}
In this paper we establish existence and uniqueness for bounded weak solutions of an elliptic-parabolic Neumann problem. We also describe the asymptotic behavior as $t \rightarrow \infty$.
\end{abstract}

1. Introduction. Let $c: \mathbf{R} \rightarrow \mathbf{R}$ be a continuous function which is strictly increasing on $\mathbf{R}^{-}$and identically equal to a constant on $\mathbf{R}^{+}$(see Figure 1 ). For a bounded domain $\Omega \subset \mathbf{R}^{n}$ with smooth boundary $\partial \Omega$ we consider the Neumann problem

(I) $\left\{\begin{array}{l}(c(u))_{t}=\Delta u \quad \text { in } Q_{T}=\Omega \times(0, T], \\ \partial u / \partial \nu=f \quad \text { on } \partial \Omega \times(0, T], \\ c(u(x, 0))=v_{0}(x), \quad x \in \Omega .\end{array}\right.$

Here $u=u(x, t), \Delta$ denotes the Laplacian in $\mathbf{R}^{n}$ and $\partial / \partial \nu$ the outward normal derivative on $\partial \Omega$. For given functions $f$ and $v_{0}$ we discuss existence, uniqueness and asymptotic behavior of (weak) solutions of Problem I. Equation (1.1) is elliptic if $u>0$ and parabolic if $u<0$. Because of this change of type we cannot a priori expect solutions to be classical.

The physical background of (1.1) lies in the theory of partially saturated flows in porous media. In that context $u$ stands for the hydrostatic potential and $c(u)$ for the moisture content or saturation. The part of $\Omega$ where $u$ is negative is called the unsaturated region, and that where $u$ is positive the saturated region. The set where $u=0$ is usually referred to as the interface or free boundary.

Equation (1.1) has been studied extensively in one space dimension. Existence and uniqueness of weak solutions were established in $[\mathbf{5}, \mathbf{7}]$ and considerable progress has been made in the description of the interface $[4,6,2]$.

If $n>1$ equation (1.1) becomes more difficult. For that situation there is the classical paper of Alt and Luckhaus [1] which deals with existence and uniqueness of weak solutions for a class of equations including (1.1). The asymptotic behavior of these solutions is described by Kröner and Rodrigues [8]. In both [1] and [8] the boundary conditions are rather general but there is one restriction: Neumann boundary conditions are excluded. It is the object of this paper to remove this restriction.

We begin by giving an existence theorem for bounded weak solutions of Problem I, making the following hypotheses about the functions $c, f$ and $v_{0}$ :

H1. $c: \mathbf{R} \rightarrow \mathbf{R}$ is uniformly Lipschitz continuous, $c \equiv 1$ on $\mathbf{R}^{+}, c \in C^{\mathbf{1}}\left(\mathbf{R}^{-}\right)$, $c^{\prime}>0$ on $\mathbf{R}^{-}$and $\lim _{s \downarrow-\infty} c(s)=0$;

Received by the editors April 29, 1986 and, in revised form, September 9, 1986.

1980 Mathematics Subject Classification (1985 Revision). Primary 35K65. 


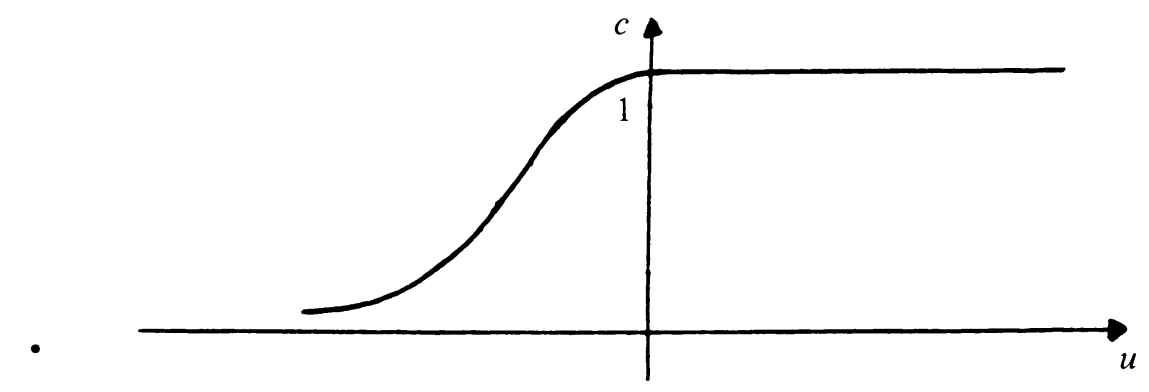

FIGURE 1. The function $c(u)$

H2. $v_{0} \in C(\Omega), v_{0} \not \equiv 1$, and there exists a function $u_{0} \in L^{\infty}(\Omega)$ such that $v_{0}=c\left(u_{0}\right)$ on $\Omega$

H3. $f \in L^{\infty}(\partial \Omega \times(0, T]), t(\partial f / \partial t) \in L^{1}(\partial \Omega \times(0, T])$ and there exists a function $g \in L^{\infty}(\partial \Omega)$ such that

$$
\begin{gathered}
\int_{\Omega} g(x) d x=0, \\
f(x, t) \geq g(x), \quad x \in \partial \Omega, 0<t \leq T ;
\end{gathered}
$$

$\mathrm{H} 4$.

$$
\int_{\Omega} v_{0}(x) d x+\int_{0}^{T} \int_{\partial \Omega} f(x, t) d x d t<|\Omega|,
$$

where $|\Omega|$ denotes the measure of $\Omega$.

DEFINITION 1.1. By a weak solution of Problem I we shall mean a function $u \in L^{2}\left(0, T ; H^{1}(\Omega)\right)$ such that

(i) $c(u) \in C\left((0, T] ; L^{2}(\Omega)\right)$;

(ii) for all test functions $\phi \in H^{1}\left(Q_{T}\right)$ with $\phi(\cdot, T)=0$ a.e. in $\Omega$ the following integral identity holds:

$$
\iint_{Q_{T}}\left\{\nabla \phi \nabla u-\phi_{t} c(u)\right\} d x d t=\int_{\Omega} \phi(x, 0) v_{0}(x) d x+\int_{0}^{T} \int_{\partial \Omega} \phi(x, t) f(x, t) d x d t .
$$

THEOREM 1.1. Let the assumptions H1-4 be satisfied. Then Problem I has a unique weak solution $u$. In addition we have

(i) $u \in L^{\infty}\left(Q_{T}\right)$ and $c(u) \in C\left(Q_{T}\right)$;

(ii) $t \nabla u(t) \in L^{\infty}\left(0, T ; L^{2}(\Omega)\right)$, and $c(u) \in H^{1}(\Omega \times[\tau, T])$ for all $\tau \in(0, T)$;

(iii) if $u_{0} \in H^{1}(\Omega)$ and $\partial f / \partial t \in L^{1}(\partial \Omega \times(0, T])$, then $u \in L^{\infty}\left(0, T ; H^{1}(\Omega)\right)$ and $c(u) \in H^{1}\left(Q_{T}\right)$.

Before we continue, we briefly discuss Theorem 1.1 in relation to the results of Alt and Luckhaus. They exclude Neumann boundary conditions essentially because they control the $H^{1}$-norm of $u$ in $\Omega$ with the $L^{2}$-norm of $\nabla u$ in $\Omega$ and the prescribed values of $u$ at some part of the lateral boundary. In dealing with the Neumann problem one has to find a substitute for this argument. 
Our alternative is the construction of a bound on the $L^{\infty}$-norm of $u$ in $\Omega$. This has the advantage that we can use a regularity result of DiBenedetto and Gariepy. In [3] they prove that for bounded weak solutions $u$ of $(1.1)$ the saturation $c(u)$ is continuous.

A lower bound is easily established because of the appearance of the function $g$ in H3. As a stationary subsolution for Problem I we can simply take a solution of

$$
\text { (II) } \begin{cases}\nabla u=0, & \Omega, \\ \partial u / \partial \nu=g, & \partial \Omega,\end{cases}
$$

and hence a lower bound is immediate.

The construction of an upper bound for $u$ will require more effort. Observe that integration of (1.1) over $Q_{t}$ leads at least formally to a conservation law:

$$
\int_{\Omega} c(u(x, t)) d x=\int_{\Omega} v_{0}(x) d x+\int_{0}^{t} \int_{\partial \Omega} f(x, s) d x d s .
$$

Because of (1.4) and (1.5) the expression on the right-hand side of (1.8) is nondecreasing in $t$. Thus assumption $\mathrm{H} 4$ merely states that $\int_{\Omega} c(u(x, t)) d x$ is bounded away from $|\Omega|$ on $[0, T]$. We conclude, still formally, that the measure of the set $\{x \in \Omega: u(x, t)<0\}$ is bounded away from zero and this will lead to an upper bound for $u$.

Once existence and uniqueness are established we turn our attention to the behavior of $u(x, t)$ as $t \rightarrow \infty$. Of course this is only possible if the assumptions hold for all $T>0$. For time independent boundary conditions this implies that

$$
\int_{\partial \Omega} f(x, t) d x=\int_{\partial \Omega} f(x) d x=0
$$

because of $\mathrm{H} 4$. Using a Liapounov argument we shall prove the following theorem.

THEOREM 1.2. Let H1-3 be satisfied and suppose that $f \equiv g$ in H3. Then the weak solution $u$ of Problem I exists for all $T>0$ and $u(\cdot, t) \rightarrow \hat{u}$ in $H^{1}(\Omega)$ as $t \rightarrow \infty$, where $\hat{u}$ is a solution of Problem II and satisfies

$$
\int_{\Omega} c(\hat{u}(x)) d x=\int_{\Omega} v_{0}(x) d x .
$$

The proof of Theorem 1.2 does not use the fact that $c(-\infty)=0$.

In the case of time dependent boundary conditions we obtain a similar but weaker result.

THEOREM 1.3. Let $\mathrm{H} 1-4$ be satisfied for all $T>0$ and suppose that $f-g \in$ $L^{1}\left(\partial \Omega \times \mathbf{R}^{+}\right)$, and that

$$
\int_{\Omega} v_{0}(x) d x+\int_{0}^{\infty} \int_{\partial \Omega} f(x, t) d x d t<|\Omega|
$$

Then the weak solution $u$ of Problem I exists for all $T>0$ and $c(u(\cdot, t)) \rightarrow c(\hat{u})$ in $L^{1}(\Omega)$ as $t \rightarrow \infty$ where $\hat{u}$ is a solution of Problem II and satisfies

$$
\int_{\Omega} c(\hat{u}(x)) d x=\int_{\Omega} v_{0}(x) d x+\int_{0}^{\infty} \int_{\partial \Omega} f(x, t) d x d t
$$

So in both cases the solution converges to the equilibrium solution which has a "total mass" prescribed by the conservation law (1.8). 
As a corollary of Theorems 1.1-1.3 and the continuity result of DiBenedetto and Gariepy

$$
c(u(\cdot, t)) \rightarrow c(\hat{u}) \quad \text { as } t \rightarrow \infty \text { in } C(K)
$$

for any compact $K \subset \Omega$.

REMARK. The lower bound on $f$, required in H3, imposed a severe restriction on the boundary condition we can allow. We can omit it, but unfortunately only at the expense of a physically unrealistic assumption on $c$. Thus Theorems 1.1-1.3 remain valid if we replace $\mathrm{H} 1, \mathrm{H} 3$ and $\mathrm{H} 4$ by

$\mathrm{H} 1^{*} . c: \mathbf{R} \rightarrow \mathbf{R}$ is uniformly Lipschitz continuous, $c \equiv 1$ on $\mathbf{R}^{+}, c \in C^{1}\left(\mathbf{R}^{-}\right)$, $c^{\prime}>0$ on $\mathbf{R}^{-}$and

$$
\liminf _{s \downarrow-\infty} c^{\prime}(s)>0
$$

$\mathrm{H}^{*} . f \in L^{\infty}(\partial \Omega \times(0, T]), t(\partial f / \partial t) \in L^{1}(\partial \Omega \times(0, T]) ;$

$\mathrm{H} 4^{*}$.

$$
\sup _{0 \leq t \leq T}\left\{\int_{\Omega} v_{0}(x) d x+\int_{0}^{t} \int_{\partial \Omega} f(x, s) d x d x\right\}<|\Omega| .
$$

Note that $\mathrm{H} 1^{*}$ implies that $c(-\infty)=-\infty$ and that $\mathrm{H} 4$ had to be revised too, because the right-hand side of (1.8) is not necessarily monotone.

An alternative approach would be not to work with $L^{\infty}$-solutions. In the first step of the existence proof however, Lemma 2.2 in the next section, we obtain an a priori bound for the $L^{2}\left(Q_{T}\right)$-norm of $u$, using the a priori $L^{\infty}$-bound for $u$. It is possible to prove Lemma 2.2 without (1.13), if one has a Poincaré inequality for $u$. This Poincaré inequality can be derived from the conversation law (1.8) and a variant of the compatibility condition (1.6). Once Lemma 2.2 is established, the remainder of $\S 2$ can be done in much the same way. We shall not go into the details of this.

2. Regularization of Problem I; properties of weak solutions. In order to construct a weak solution of Problem I we use a parabolic regularization. We approximate the functions $c, u_{0}$ and $f$ by sequences of smooth functions $c_{n}, u_{0 n}$ and $f_{n}, n=1,2, \ldots$, which have the following properties:

(i) There exist constants $K, L, L_{1}$ and $M$ such that for all $n \in \mathrm{N}$

$$
\begin{gathered}
1 / n \leq c_{n}^{\prime} \leq K \quad \text { on } \mathbf{R}, \\
\left|u_{0 n}\right| \leq M \quad \text { on } \bar{\Omega}, \\
\left|f_{n}\right| \leq L \quad \text { on } \partial \Omega \times[0, T], \\
\left\|t\left(\partial f_{n} / \partial t\right)\right\|_{L^{1}(\partial \Omega \times(0, T))} \leq L_{1} .
\end{gathered}
$$

In addition, if $u_{0} \in H^{1}(\Omega)$ and $\partial f / \partial t \in L^{1}(\partial \Omega \times(0, T))$, there exist constants $L_{2}$ and $L_{3}$ such that for all $n \in \mathbf{N}$

$$
\begin{gathered}
\left\|u_{0 n}\right\|_{H^{1}(\Omega)} \leq L_{2}, \\
\left\|\partial f_{n} / \partial t\right\|_{L^{1}(\partial \Omega \times(0, T))} \leq L_{3} .
\end{gathered}
$$

$$
\begin{gathered}
f_{n} \geq g \quad \text { a.e. on } \partial \Omega \times(0, T], \\
\partial u_{0 n}(x) / \partial \nu=f_{n}(x, 0), \quad x \in \partial \Omega
\end{gathered}
$$


(iii)

$$
\begin{gathered}
c_{n} \rightarrow c \quad \text { in } L_{\mathrm{loc}}^{\infty}(\mathbf{R}), W_{\mathrm{loc}}^{1, \infty}\left(\mathbf{R}^{-}\right) \quad \text { and } \quad W_{\mathrm{loc}}^{1, \infty}\left(\mathbf{R}^{+}\right) \\
c_{n}\left(u_{0 n}\right) \rightarrow v_{0} \quad \text { in } L_{\mathrm{loc}}^{\infty}(\Omega) \text { and } L^{1}(\Omega) \\
f_{n} \rightarrow f \quad \text { in } L^{1}(\partial \Omega \times(0, T)) .
\end{gathered}
$$

From [9] we know that there exists a unique classical solution $u_{n}$ of the following uniformly parabolic problem:

$$
\left\{\begin{array}{l}
c_{n}^{\prime}(u) u_{t}=\Delta u \quad \text { in } Q_{T} \\
\partial u / \partial \nu=f_{n} \quad \text { on } \partial \Omega \times(0, T] \\
u(x, 0)=u_{0}(x), \quad x \in \bar{\Omega} .
\end{array}\right.
$$

We shall show that there exists a subsequence $u_{\mu}$ which converges to a weak solution of Problem I. In this section we prove this, assuming that we have a uniform $L^{\infty}$. bound on $u_{n}$. The main difficulty however, is to obtain such a bound. This will be done in the next section.

PROPOSITION 2.1. Suppose that the sequence $\left\{u_{n}\right\}$ is bounded in $L^{\infty}\left(Q_{T}\right)$ by a constant $M_{1}$. Then there exists a weak solution $u$ of Problem $\mathrm{I}$.

Before giving the proof we derive some estimates on the solutions $u_{n}$. By $\|\cdot\|_{\infty}$ we shall always mean the norm in $L^{\infty}\left(Q_{T}\right)$.

LEMMA 2.2. Suppose $\left\|u_{n}\right\|_{\infty} \leq M_{1}$. Then there exists a constant $C_{1}$, which does not depend on $n$, such that for all $n$

$$
\iint_{Q_{T}}\left|\nabla u_{n}\right|^{2} \leq C_{1}
$$

ProOF. Define $q_{n}: \mathbf{R} \rightarrow \overline{\mathbf{R}}^{+}$by

$$
q_{n}(s)=\int_{0}^{s} \sigma c_{n}^{\prime}(\sigma) d \sigma=\int_{0}^{s}\left\{c_{n}(s)-c_{n}(\sigma)\right\} d \sigma .
$$

If we multiply the equation for $u_{n}$ by $u_{n}$ and integrate by parts we obtain, omitting the subscript $n$,

$$
\int_{\Omega} q(u(x, T)) d x+\iint_{Q_{T}}|\nabla u|^{2}=\int_{0}^{T} \int_{\partial \Omega} u f+\int_{\Omega} q\left(u_{0}(x)\right) d x .
$$

Since the right-hand side of (2.14) is bounded by

$$
C_{1} \stackrel{\text { def }}{=} M_{1} L T|\partial \Omega|+\frac{1}{2} K M^{2}|\Omega|
$$

where $K, M$ and $L$ are as in (2.1), (2.2) and (2.3), (2.12) follows.

LEMMA 2.3. Suppose $\left\|u_{n}\right\|_{\infty} \leq M_{1}$. Then there exist constants $C_{2}$ and $C_{3}$, which do not depend on $n$, such that for all $n$

$$
\iint_{Q_{T}} t\left\{\left(c_{n}\left(u_{n}\right)\right)_{t}\right\}^{2} \leq C_{2}
$$

and

$$
\int_{\Omega}\left|\nabla u_{n}(x, t)\right|^{2} d x \leq \frac{C_{3}}{t}, \quad 0<t \leq T
$$


PROOF. We multiply the equation for $u_{n}$ by $t u_{n t}$ and obtain, integrating by parts over $Q_{\tau}(0 \leq \tau \leq T)$ and omitting the subscript $n$

$$
\begin{aligned}
& \iint_{Q_{\tau}} t c^{\prime}(u) u_{t}^{2}+\frac{\tau}{2} \int_{\Omega}|\nabla u(x, \tau)|^{2} d x \\
& \quad=\tau \int_{\partial \Omega} f(x, \tau) u(x, \tau) d x-\int_{0}^{\tau} \int_{\partial \Omega}\left(t f_{t}+f\right) u+\frac{1}{2} \iint_{Q_{\tau}}|\nabla u|^{2} .
\end{aligned}
$$

The right-hand side of $(2.18)$ is bounded by

$$
C \stackrel{\text { def }}{=} 2 M_{1} L T|\partial \Omega|+M_{1} L_{1}+\frac{1}{2} C_{1},
$$

where $L$ and $L_{1}$ are as in (2.3) and (2.4). If we put $C_{2}=K C$ and $C_{3}=2 C,(2.16)$ and (2.17) follow.

REMARK. If $u_{0} \in H^{1}(\Omega)$ and $\partial f / \partial t \in L^{1}(\partial \Omega \times(0, T))$, then multiplying the equation by $u_{t}$ leads to an estimate of the form

$$
\iint_{Q_{t}} c_{n}^{\prime}\left(u_{n t}\right)\left(u_{n t}\right)^{2}+\frac{1}{2} \int_{\Omega}\left|\nabla u_{n}(x, t)\right|^{2} d x \leq C_{4},
$$

for some constant $C_{4}$.

PROOF OF PROPOSITION 2.1. It follows from (2.12) and (2.17) that

$$
u_{n} \text { is bounded in } L^{2}\left(0, T ; H^{1}(\Omega)\right),
$$

and that for any $\tau \in(0, T)$

$$
u_{n} \text { is bounded in } L^{\infty}\left(\tau, T ; H^{1}(\Omega)\right) .
$$

Hence, by (2.16),

$$
v_{n} \stackrel{\text { def }}{=} c_{n}\left(u_{n}\right) \text { is bounded in } H^{1}(\Omega \times(\tau, T)) .
$$

Consequently there exists a subsequence which we also denote by $\left\{u_{n}\right\}$, such that

$$
\begin{array}{ll}
u_{n} \rightarrow u & \text { in } L^{2}\left(0, T ; H^{1}(\Omega)\right), \\
u_{n} \stackrel{*}{\rightarrow} u & \text { in } L^{\infty}\left(\tau, T ; H^{1}(\Omega)\right)
\end{array}
$$

for any $\tau \in(0, T)$,

$$
-\|u\|_{\infty} \leq M_{1} \text {, and }
$$

$$
\begin{array}{ll}
v_{n} \stackrel{*}{*} v & \text { in } L^{\infty}\left(Q_{T}\right), \\
v_{n} \rightarrow v & \text { in } H^{1}(\Omega \times(\tau, T)) .
\end{array}
$$

We claim that $v=c(u)$. To see this observe that

$$
\left\|c_{n}\left(u_{n}\right)-c\left(u_{n}\right)\right\|_{\infty} \leq \max _{|s| \leq M_{1}}\left|c_{n}(s)-c(s)\right| \rightarrow 0 .
$$

Combining this with $(2.21)$ and the compact imbedding $H^{1} \hookrightarrow L^{2}$ we obtain $c\left(u_{n}\right) \rightarrow v$ in $L^{2}(\Omega \times(\tau, T))$. From $(2.20)$ we also know that $u_{n} \rightarrow u$ in $L^{2}(\Omega \times(\tau, T))$, and thus we may conclude that $v=c(u)$. It is easily checked that $u$ is a weak solution of Problem I.

REMARK. If $u_{0} \in H^{1}(\Omega)$ and $\partial f / \partial t \in L^{1}(\partial \Omega \times(0, T))$ we have

$$
u_{n} \stackrel{*}{\rightarrow} u \quad \text { in } L^{\infty}\left(0, T ; H^{1}(\Omega)\right)
$$


and

$$
c_{n}\left(u_{n}\right) \rightarrow c(u) \quad \text { in } H^{1}\left(Q_{T}\right) .
$$

COROLlARY 2.4. Let $u$ be the weak solution as constructed in Proposition 2.1. Then $u \in L^{\infty}\left(Q_{T}\right), t u(x, t) \in L^{\infty}\left(0, T ; H^{1}(\Omega)\right)$ and $c(u) \in H^{1}(\Omega \times(\tau, T))$ for all $\tau \in(0, T)$. If $u_{0} \in H^{1}(\Omega)$ and $\partial f / \partial t \in L^{1}(\partial \Omega \times(0, T))$, then $u \in L^{\infty}\left(0, T ; H^{1}(\Omega)\right)$ and $c(u) \in H^{1}\left(Q_{T}\right)$. Furthermore

$$
\begin{array}{r}
\iint_{Q_{t}}\left\{\nabla \phi \nabla u-\phi_{t} c(u)\right\} d x d t+\int_{\Omega} \phi(x, T) c(u(x, T)) d x \\
=\int_{\Omega} \phi(x, 0) v_{0}(x) d x+\int_{0}^{T} \int_{\partial \Omega} \phi(x, t) f(x, t) d x d t
\end{array}
$$

for all $\phi \in H^{1}\left(Q_{T}\right)$.

Thus we know that Problem I has a bounded weak solution $u$, provided that the sequence $\left\{u_{n}\right\}$ is uniformly bounded in $L^{\infty}\left(Q_{T}\right)$. Concerning the regularity of the weak solution we recall a result of DiBenedetto and Gariepy.

THEOREM 2.5. Let $u$ be a bounded weak solution of Problem I on $Q_{T}$ and let $K \subset Q_{T}$ be compact. Then $c(u)$ is continuous on $K$, and the modulus of continuity depends only on the dimension $n$, the shape of the function $c$, the $L^{\infty}\left(Q_{T}\right)$-norm of $u$, and the distance of $K$ to the parabolic boundary $\Gamma_{T}$ of $Q_{T}$. In particular $c(u) \in C\left(Q_{T}\right)$.

We conclude this section with two propositions.

PROPOSITION 2.6. Problem I has at most one weak solution.

The proof of Proposition 2.6 is identical to the uniqueness proof in [5] and therefore we omit it.

PROPOSITION 2.7. Any weak solution $u$ of Problem I satisfies the conservation law (1.8) for any $t \in(0, T]$.

ProOF. We substitute the test function $\phi(x, t)=(\tau-t)^{+}(0 \leq \tau \leq T)$ into (1.7) and differentiate with respect to $\tau$. This yields (1.8).

3. Local existence of bounded solutions. In order to prove existence of a weak solution we still have to find lower and upper bounds for the classical solutions of the regularized problems $\left(\mathrm{I}_{n}\right)$. We first give a global lower bound and then a local upper bound.

PROPOSITION 3.1. There exists a function $\underline{u} \in C(\bar{\Omega})$, such that $u_{n} \geq \underline{u}$ on $Q_{T}$ for all $n \in \mathbf{N}$.

ProOF. Let $w \in H^{1}(\Omega)$ be the solution of Problem II which satisfies

$$
\int_{\Omega} w(x) d x=0 \text {. }
$$

Then $w$ is uniquely determined and from standard regularity theory we know that $w \in W^{1, p}(\Omega)$ for all $1 \leq p<\infty$. Hence $w \in C(\bar{\Omega})$ and the function $\underline{u} \in C(\bar{\Omega})$, defined by $\underline{u}=w-\|w\|_{\infty}-M$, where $M$ is as in (2.2), is a subsolution for all the problems $\left(\mathrm{I}_{n}\right)$. Consequently $\underline{u} \leq u_{n}$ on $Q_{T}$ for all $n$.

The following proposition is the main result of this section and establishes a local upper bound. 
PROPOSITION 3.2. Suppose that for some $\delta>0$

$$
\int_{\Omega} v_{0}(x) d x \leq(1-\delta)|\Omega|
$$

Then there exist constants $\bar{M}>0$ and $\tau>0$, and a function $\bar{u}(x, t)$ on $Q_{\tau}$ such that $\bar{u}$ is a supersolution for all the regularized problems $\left(\mathrm{I}_{n}\right)$ and $\bar{u} \leq \bar{M}$. The constant $\bar{M}$ depends only on $\Omega, L$ and $\delta$, where $L$ is as in (2.3).

REMARK. This proposition will enable us to continue bounded local solutions. Note that the constant $\tau$ may (and will) depend on the function $v_{0}$. To prove Proposition 3.2 we need some lemmas.

LEMMA 3.3. There exist positive constants $\eta_{1}, \eta_{2}$ and $\eta_{3}$, depending only on $\Omega$ and the constant $\delta$ appearing in (3.1), and a finite number of disjunct closed balls $B_{1}, \ldots, B_{m} \subset \Omega$ such that

$$
\begin{gathered}
\sum_{i=1}^{m}\left|B_{i}\right| \geq \eta_{1}, \\
\operatorname{dist}\left(B_{i}, \partial \Omega\right) \geq \eta_{2}, \quad i=1, \ldots, m, \\
v_{0} \leq 1-\eta_{3} \quad \text { on } \bigcup_{i=1}^{m} B_{i} .
\end{gathered}
$$

PROOF. This follows from the continuity of $v_{0}$ on $\Omega$, from the fact that $0 \leq$ $v_{0} \leq 1$ on $\Omega$ and from (3.1). We leave the details to the reader.

LEMMA 3.4. For every closed ball $B_{i}$ as in Lemma 3.3 , let $\tilde{B}_{i}$ be a concentric ball with $\left|\tilde{B}_{i}\right|=\frac{1}{2}\left|B_{i}\right|$. Let $w$ be the unique classical solution of the problem

$$
\begin{cases}\Delta w=0 & \text { in } \Omega \backslash\left(\bigcup_{i=1}^{m} \tilde{B}_{i}\right), \\ w=0 & \text { on } \bigcup_{i=1}^{m} \partial \tilde{B}_{i}, \\ \partial w / \partial \nu=L & \text { on } \partial \Omega,\end{cases}
$$

where $L$ is as in (2.3). Then there exists a constant $\bar{M}>0$, depending only on $\Omega, L$ and the constant $\delta>0$ in (3.1), such that $0 \leq w \leq \bar{M}$ on $\Omega \backslash\left(\bigcup_{i=1}^{m} \tilde{B}_{i}\right)$.

ProOF. We shall compute an explicit bounded supersolution for Problem III.

Let $G(x, y)$ be a Green function such that for every $y \in \Omega$

$$
\begin{gathered}
G(\cdot, y)=0 \quad \text { on } \partial \Omega, \\
\Delta G(\cdot, y)=\delta(y),
\end{gathered}
$$

where $\delta(y)$ denotes the Dirac measure with unit mass in $y$.

Then, there exists a constant $\mu>0$, depending only on $\eta_{2}$, such that if $\operatorname{dist}(y, \partial \Omega) \geq \eta_{2}$,

$$
\partial G(\cdot, y) / \partial \nu \geq \mu \quad \text { on } \partial \Omega
$$

Now define $\tilde{w}(x)$ by

$$
\tilde{w}(x)=\int_{\bigcup_{i=1}^{m} \tilde{B}_{i}} G(x, y) d y .
$$




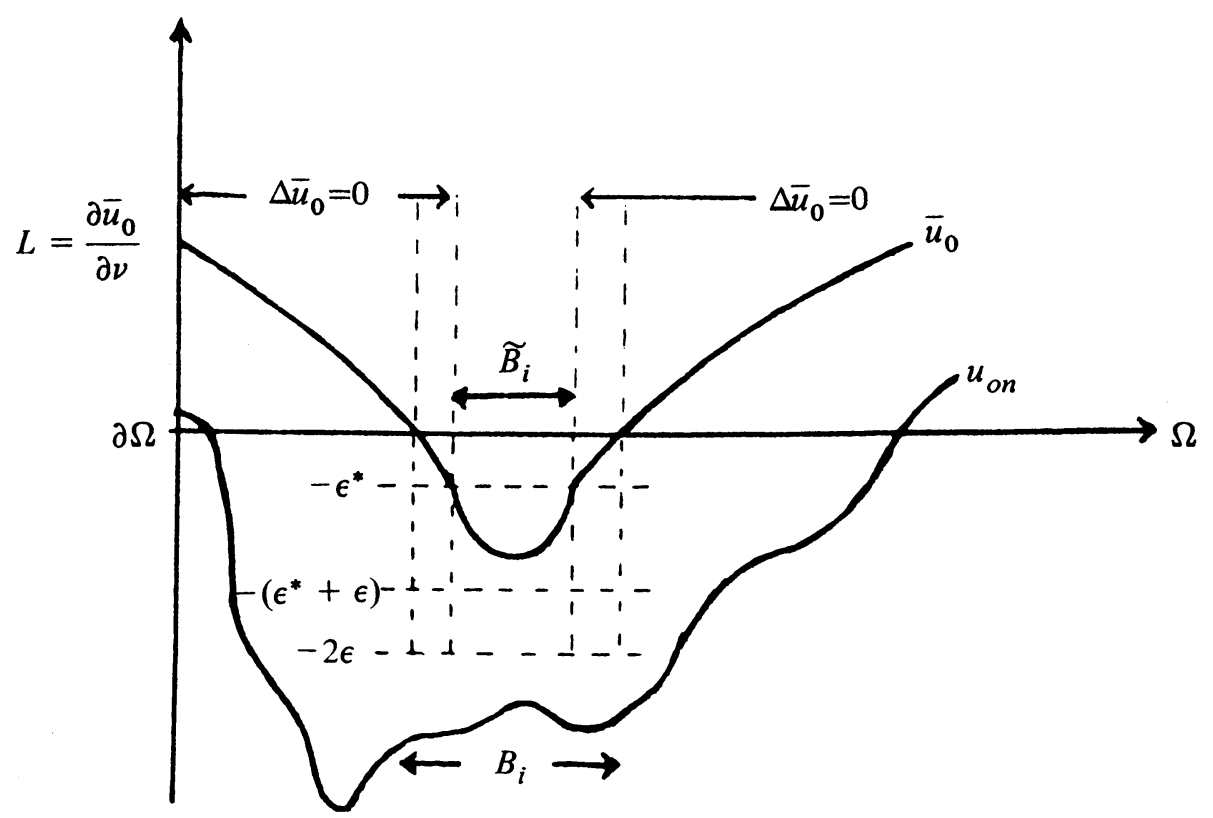

FIGURE 2. The function $\bar{u}_{0}$

Then $\tilde{w}$ satisfies

$$
\Delta \tilde{w}=0 \quad \text { in } \Omega \backslash\left(\bigcup_{i=1}^{m} \tilde{B}_{i}\right)
$$

and

$$
\frac{\partial \tilde{w}}{\partial \nu} \geq \mu \cdot \sum_{i=1}^{m}\left|\tilde{B}_{i}\right| \geq \frac{\mu \eta_{1}}{2} \quad \text { on } \partial \Omega .
$$

Furthermore we know that

$$
\tilde{u}(x) \stackrel{\text { def }}{=} \int_{\Omega} G(x, y) d y \leq \tilde{w}(x) \leq 0, \quad x \in \Omega,
$$

where $\tilde{u}$ is the classical solution of

$$
\begin{cases}\Delta u=1 & \text { in } \Omega \\ u=0 & \text { on } \partial \Omega .\end{cases}
$$

Now we can define a supersolution $\bar{w}$ for Problem III, namely

$$
\bar{w}(x)=\frac{2 L}{\mu \eta_{1}}\left\{\tilde{w}(x)-\min _{\Omega} \tilde{u}\right\} .
$$

Since $\bar{w} \geq 0$ on $\Omega$, it follows from (3.9) that $\bar{w}$ is indeed a supersolution and that

$$
0 \leq w(x) \leq \bar{w}(x), \quad x \in \Omega \backslash\left(\bigcup_{i=1}^{m} \tilde{B}_{i}\right) .
$$

Moreover

$$
\bar{w}(x) \leq \bar{M} \stackrel{\text { def }}{=}-\frac{2 L}{\mu \eta_{1}} \min \tilde{u},
$$


where $\bar{M}$ depends only on $\eta_{1}, \eta_{2}, L$ and $\Omega$. Since $\eta_{1}$ and $\eta_{2}$ only depend on $\Omega$ and $\delta$, this completes the proof of Lemma 3.4.

PROOF OF PROPOSITION 3.2. We are now ready to construct $\bar{u}$. We extend the solution $w$ of Problem III as a $H^{1}$-function to the whole of $\Omega$ in such a way that $\Delta w$ is bounded from above in the sense of distributions. To do this define $\varepsilon>0$ by

$$
c(-2 \varepsilon)=1-\eta_{3} .
$$

For every $\tilde{B}_{i}$ we choose a radially symmetric function $w_{i} \in C^{2}\left(\overline{\tilde{B}}_{i}\right)$ such that

$$
\begin{gathered}
-\varepsilon \leq w_{i} \leq 0 \quad \text { in } \tilde{B}_{i} \\
w_{i}=0 \quad \text { on } \partial \tilde{B}_{i} \\
\partial w / \partial \nu_{i} \leq \partial w_{i} / \partial \nu_{i} \quad \text { on } \partial \tilde{B}_{i},
\end{gathered}
$$

where $\nu_{i}$ is the outwards directed normal on $\partial \tilde{B}_{i}$. Now extend $w$ to $\Omega$ by

$$
w(x)=w_{i}(x), \quad x \in \tilde{B}_{i}, i=1, \ldots, m .
$$

Let $\tilde{M}=\max \left\{\Delta w_{i}(x): x \in \overline{\tilde{B}}_{i}, i=1, \ldots, m\right\}$. Then it follows from (3.15) that

$$
\Delta w \leq \tilde{M} \quad \text { in } \Omega
$$

in the sense of distributions.

We choose $\varepsilon^{*} \in(0, \varepsilon)$ such that (see Figure 2)

$$
\bar{u}_{0}(x) \stackrel{\text { def }}{=} w(x)-\varepsilon^{*}>0 \quad \text { for } x \in \partial B_{i}, i=1, \ldots, m .
$$

By (3.4), (3.12) and (3.13) we have

$$
\bar{u}_{0}>u_{0} \quad \text { on } \bigcup_{i=1}^{m} B_{i},
$$

and since $\bar{u}_{0}>0$ on $\Omega \backslash\left(\bigcup_{i=1}^{m} B_{i}\right)$, it follows that the functions $u_{0 n}$ in $\S 2$ can be chosen in such a way that

$$
u_{0 n}<\bar{u}_{0} \quad \text { on } \bar{\Omega} \text {. }
$$

Choose a constant $m_{1}>0$ such that

$$
c_{n}^{\prime} \geq m_{1} \quad \text { on }\left[-\varepsilon,-\frac{1}{2} \varepsilon^{*}\right]
$$

for all $n \in \mathbf{N}$ and define

$$
\bar{u}(x, t)=\bar{u}_{0}(x)+\left(\tilde{M} / m_{1}\right) t, \quad x \in \bar{\Omega}, t \geq 0 .
$$

We claim that $\bar{u}$ is a supersolution for Problem $\mathrm{I}_{n}$ on $Q_{\tau}$, where

$$
\tau=\varepsilon^{*} m_{1} / 2 \tilde{M}
$$

To check this we observe that

$$
c_{n}^{\prime}(\bar{u}) \bar{u}_{t}=c_{n}^{\prime}(\bar{u}) \tilde{M} / m_{1} \geq \tilde{M} \geq \Delta \bar{u}
$$

on $\tilde{B}_{i} \times(0, \tau], i=1, \ldots, m$, and that

$$
\Delta \bar{u}_{0}=0 \quad \text { in } \Omega \backslash\left(\bigcup_{i=1}^{m} \tilde{B}_{i}\right) .
$$


Furthermore, for all $n \in \mathbf{N}$,

$$
\partial \bar{u} / \partial \nu=L \geq \partial u_{n} / \partial \nu \quad \text { on } \partial \Omega \times(0, T] .
$$

Combining this with (3.20) we can conclude, by means of the classical comparison principle for parabolic equations, that

$$
\bar{u} \geq u_{n} \quad \text { on } Q_{\tau} \quad \text { for all } n \in \mathbf{N} .
$$

Since $\bar{u} \leq w \leq \bar{M}$ on $Q_{\tau}$, where $\bar{M}$ is defined by (3.11), this completes the proof of Proposition 3.2.

A direct consequence of Propositions 2.1, 3.1 and 3.2 is the following proposition.

PROPOSITION 3.5 (LOCAL EXISTENCE OF BOUNDED SOLUTIONS). There exists a $\tau>0$ such that Problem I has a unique weak solution $u$ on $Q_{\tau}$ which has the regularity properties described in Corollary 2.4 and which satisfies

$$
\underline{u} \leq u \leq \bar{M} \text {, }
$$

on $Q_{\tau}$, where $\underline{u} \in C(\bar{\Omega})$ is the subsolution appearing in Proposition 3.1 and $\bar{M}$ is defined by (3.11). The constant $\bar{M}$ depends only on $\Omega, L$ and $\delta$.

REMARK. One could hope to prove Theorem 1.1 by applying Proposition 3.5 to continue the solution in a finite number of timesteps to the whole of $Q_{T}$. However, since we do not control $\tau$, which depends on $v_{0}$, this approach fails. The timesteps may become smaller and smaller and we may never reach $T$.

4. Global existence of bounded solutions. In this section we complete the proof of Theorem 1.1 and give a comparison principle.

We define $\delta>0$ by

$$
\int_{\Omega} v_{0}(x) d x+\int_{0}^{T} \int_{\partial \Omega} f(x, t) d x d t=(1-\delta) \cdot|\Omega| .
$$

From the results in $\S 3$, we know that there exists $\tau>0$ and $\bar{M}>0$ such that Problem I has a unique weak solution $u$ on $Q_{\tau}$, which satisfies

$$
\underline{u} \leq u \leq \bar{M}
$$

on $Q_{\tau}$. Here $\underline{u}$ is the stationary subsolution appearing in Proposition 3.1. By Proposition 3.2 , the constant $\bar{M}$ depends only on the constant $\delta$ as defined in (4.1). We now define $T^{*}$ by

$$
\begin{array}{r}
T^{*}=\sup \left\{0<t_{1} \leq T:\right. \text { Problem I has a unique weak } \\
\text { solution on } \left.Q_{t_{1}} \text { which satisfies (4.2) }\right\} .
\end{array}
$$

Because of the uniqueness of weak solutions all these solutions can be seen as restrictions of one function in $L^{\infty}\left(Q_{T^{*}}\right)$. There will be no confusion if we denote this function again by $u$.

What we have to show is that $u=u(t)$ is not only a solution of Problem I on $\left[0, T^{*}\right)$, but even on $\left[0, T^{*}\right]$, and that $T^{*}=T$. Clearly $T^{*} \geq \tau>0$. Observe, however, that $u$ is only on $Q_{\tau}$ the limit of the approximating solutions $u_{n}$. At this point we do not know anything about the regularity of $u$ for $t>\tau$, apart from the continuity of $c(u)$ (Theorem 2.5). The following lemma clarifies the situation for $\tau<t \leq T^{*}$. 
LEMMA 4.1. Let $T^{*}$ be defined by (4.3). Then $u$ is a weak solution of Problem I on $Q_{T^{*}}$ and it has the regularity properties described in Corollary 2.4 and Theorem 2.5. In particular, for all test functions $\phi \in H^{1}\left(Q_{T^{*}}\right)$

$$
\begin{gathered}
\iint_{Q_{T^{*}}}\left\{\nabla_{\phi} \nabla u-\phi_{t} c(u)\right\} d x d t+\int_{\Omega} \phi\left(x, T^{*}\right) c\left(u\left(x, T^{*}\right)\right) d x \\
=\int_{\Omega} \phi(x, 0) v_{0}(x) d x+\int_{0}^{T^{*}} \int_{\partial \Omega} \phi(x, t) f(x, t) d x d t .
\end{gathered}
$$

Leaving the proof of Lemma 4.1 aside for the moment, we first finish the proof of Theorem 1.1.

PROOF OF THEOREM 1.1. Because of Lemma 4.1 it is sufficient to prove that $T^{*}=T$. We argue by contradiction and assume that $T^{*}<T$.

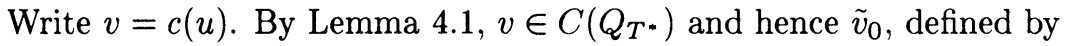

$$
\tilde{v}_{0}=v\left(\cdot, T^{*}\right) \text {, }
$$

is well defined and $\tilde{v}_{0} \in C(\Omega)$. Since, for almost all $t \in\left(0, T^{*}\right), v(\cdot, t)=c(u(\cdot, t))$ and, again by Lemma 4.1, $u(\cdot, t)$ is bounded in $H^{1}(\Omega)$ as $t \uparrow T^{*}$, $\tilde{v}_{0}$ satisfies $\mathrm{H} 2$. By (4.1) and Proposition 2.7 it also satisfies (3.1). Thus we can apply the local existence theory as developed in $\S 3$ to obtain a bounded local weak solution for $t>T^{*}$. More precisely, we find a time $\tilde{\tau}>T^{*}$ and a function

$$
\tilde{u} \in L^{\infty}\left(\Omega \times\left(T^{*}, \tilde{\tau}\right)\right) \cap L^{\infty}\left(T^{*}, \tilde{\tau} ; H^{1}(\Omega)\right),
$$

such that $c(\tilde{u})_{t} \in L^{2}\left(\Omega \times\left(T^{*}, \tilde{\tau}\right)\right)$, and such that for all $\phi \in H^{1}\left(\Omega \times\left(T^{*}, \tilde{\tau}\right)\right)$ vanishing for $t=\tilde{\tau}$, the following integral identity holds:

$$
\int_{T^{*}}^{\tilde{\tau}}\left\{\nabla \phi \nabla u-\phi_{t} c(u)\right\} d x d t=\int_{\Omega} \phi\left(x, T^{*}\right) \tilde{v}_{0}(x) d x+\int_{T^{*}}^{\tilde{\tau}} \int_{\partial \Omega} \phi(x, t) f(x, t) d x d t .
$$

Note that for the construction of a bound on $\tilde{u}$ we can take the same subsolution $u$ and the same constant $\bar{M}$ as in $\S 3$. By Proposition 3.5 we know that $\underline{u} \leq \tilde{u} \leq \bar{M}$ on $\Omega \times\left(T^{*}, \tilde{\tau}\right]$. We now extend $u$ to $Q_{\tilde{\tau}}$ by setting $u=\tilde{u}$ on $\Omega \times\left(T^{*}, \tilde{\tau}\right]$. It follows from (4.4) and (4.6) that $u$ is a weak solution on $Q_{\tilde{\tau}}$ which satisfies (4.2). This contradicts the definition of $T^{*}$ and thus $T^{*}=T$. Theorem 1.1 now follows from Lemma 4.1.

PROOF OF LEMMA 4.1. The idea of the proof is to reconstruct the solution with the techniques developed in $\S 3$ but with a slightly different supersolution. All we have to do is to get a hold on the timestep $\tau$ in Proposition 3.2. This would allow us to carry out successfully the approach sketched at the end of $\S 4$.

The reason why we do not control the timestep $\tau$ in Proposition 3.2 is that we do not control the radii of the balls $B_{1}, \ldots, B_{m}$ in Lemma 3.3. Thus we need a variant of Lemma 3.3 .

LEMMA 4.2. There exist numbers $R>0, \tilde{\eta}_{2}>0$ and $\tilde{\eta}_{3}>0$ such that for every $t \in\left[\tau, T^{*}\right)$ a closed ball $B(t)$ with radius $R$ and $\operatorname{dist}(B(t), \partial \Omega) \geq \tilde{\eta}_{2}$ exists on which $c(u(\cdot, t)) \geq 1-\tilde{\eta}_{3}$.

ProOF. We use Theorem 2.5. Since $u \in L^{\infty}\left(Q_{T^{*}}\right)$, Theorem 2.5 states that $c(u)$ is continuous in $\Omega \times\left[\tau, t_{1}\right]$ for any $t_{1} \in\left[\tau, T^{*}\right)$ and that the modulus of continuity 
depends only on the distance to $\partial \Omega \times\left[\tau, T^{*}\right)$ and not on $t_{1}$. Since $0<c(u(\cdot, t)) \leq 1$ on $\Omega$ and since by $(4.1)$

$$
\int_{\Omega} c(u(x, t)) d x \leq(1-\delta) \cdot|\Omega|,
$$

the existence of $R, \tilde{\eta}_{2}$ and $\tilde{\eta}_{3}$ follows. The details are left to the reader.

In the construction of the supersolution in $\S 3$ we can now replace $\eta_{1}, \eta_{2}$ and $\eta_{3}$ by respectively $\tilde{\eta}_{1} \stackrel{\text { def }}{=}|B(t)|, \tilde{\eta}_{2}$ and $\tilde{\eta}_{3}$, and the collection of balls $B_{1}, \ldots, B_{m}$ by the single ball $B(t)$. Then the constant $\tau$, defined by (3.23), will depend on $\eta_{1}, \eta_{2}$ and $\eta_{3}$, on $L, c$ and $\Omega$, but apart from that only on the center of the ball $B(t)$. Since this center varies over a compact set and since all the constructions can be done in such a way that the dependence of $\tau$ on the center of $B(t)$ is continuous, it follows that $\tau$ can be estimated from below by a positive constant $\tau^{*}$.

Now we are able to reconstruct $u$ in a finite number of timesteps and Lemma 4.1 follows.

We conclude this section with a comparison principle.

THEOREM 4.3. Suppose that H1-4 hold for two sets of boundary conditions $v_{0}, f$ and $\tilde{v}_{0}, \tilde{f}$. If $v_{0} \leq \tilde{v}_{0}$ in $\Omega$ and $f \leq \tilde{f}$ on $[0, T]$ a.e. then the corresponding solutions also have the property that $u \leq \tilde{u}$ a.e. in $Q_{T}$.

PROOF. Because of the $L^{\infty}$-bounds on $u$ and $\tilde{u}$ we can reconstruct $u$ and $\tilde{u}$ simultaneously in a finite number of timesteps. In every timestep the functions $u_{0 n}, f_{n}$ and $\tilde{u}_{0 n}, \tilde{f}_{n}$ in $\S 2$ can be chosen in such a way that $u_{0 n} \leq \tilde{u}_{0 n}$ and $f_{n} \leq \tilde{f}_{n}$. The classical comparison principle implies that $u_{n} \leq \tilde{u}_{n}$. Since $u_{n}-\tilde{u}_{n} \rightarrow u-\tilde{u}$ in $L^{2}$, it follows that $u-\tilde{u} \leq 0$ a.e.

5. Asymptotic behavior for the autonomous problem. In this section we prove Theorem 1.2, where we assume that H1-3 hold with $f \equiv g$. Without loss of generality we take $u_{0} \in H^{1}(\Omega)$. Observe that in this case the existence proof can be simplified substanteously. The functions $f_{n}$ in $\S 2$ can be taken to be time independent and to satisfy

$$
\int_{\partial \Omega} f_{n}(x) d x=0
$$

and

$$
f_{n} \rightarrow f \quad \text { in } L^{p}(\delta \Omega) \text { for all } p \geq 1
$$

Using solutions of

$$
\begin{cases}\Delta u=0 & \text { in } \Omega, \\ \partial u / \partial \nu=f_{n} & \text { on } \partial \Omega,\end{cases}
$$

as sub- and supersolutions it is now not difficult to construct a global and uniform $L^{\infty}$-bound for the approximating solutions $u_{n}$, independent of $T$. Multiplying the equation for $u_{n}$ by $u_{n t}$ and integrating by parts, a uniform estimate for $\left\|u_{n}(\cdot, t)\right\|_{H^{1}(\Omega)}$ independent of $n$ and $t$ follows immediately. Consequently (2.20) and (2.21) hold for any $T>0$. 
To study the asymptotic behavior of $u$ we use a Liapounov function argument. We define $q: \mathbf{R} \rightarrow \mathbf{R}$ by

$$
q(s)=\int_{0}^{s} \sigma c^{\prime}(\sigma) d \sigma=\int_{0}^{s}\{c(s)-c(\sigma)\} d \sigma
$$

and $V:[0, \infty) \rightarrow \mathbf{R}$ by

$$
V(t)=\int_{\Omega}\{q(u(x, t))-\hat{u}(x) c(u(x, t))\} d x,
$$

where $\hat{u}$ is the asymptotic solution appearing in Theorem 1.2. Observe that $q$ only depends on $c(s)$ and that $q(s)=0$ for $s \geq 0$.

If we differentiate (6.4) two times, we obtain, after a formal calculation, that

$$
V^{\prime}(t)=-\int_{\Omega}|\nabla u(x, t)-\nabla \hat{u}(x)|^{2} d x \leq 0
$$

and

$$
\begin{aligned}
V^{\prime \prime}(t) & =-2 \int_{\Omega}(\nabla u-\nabla \hat{u}) \nabla u_{t} d x \\
& =2 \int_{\Omega} \Delta u u_{t} d x=2 \int_{\Omega} c^{\prime}(u(x, t)) u_{t}(x, t)^{2} d x \geq 0
\end{aligned}
$$

Unfortunately, we do not have a rigorous proof of (5.5) and (5.6). However, the following lemma will be sufficient for our purposes.

LEMMA 5.1. Let $V$ be defined by (6.4). Then $V$ is convex on $[0, \infty)$ and

$$
V\left(t_{2}\right) \leq V\left(t_{1}\right)-\int_{t_{1}}^{t_{2}} \int_{\Omega}|\nabla u(x, t)-\nabla \hat{u}(x)|^{2} d x d t
$$

for all $0 \leq t_{1} \leq t_{2}<\infty$. Thus $V$ is a Liapounov function.

PROOF OF LEMMA 5.1. We return to the classical solution $u_{n}$ of the regularized problem. Define $q_{n}$ by $(2.13)$ and $V_{n}$ by

$$
V_{n}(t)=\int_{\Omega}\left\{q_{n}\left(u_{n}(x, t)\right)-\hat{u}_{n}(x) c_{n}\left(u_{n}(x, t)\right)\right\} d x
$$

where $\hat{u}_{n}$ is the solution of Problem $\mathrm{II}_{n}$ which satisfies

$$
\int_{\Omega} c_{n}\left(\hat{u}_{n}(x)\right) d x=\int_{\Omega} c_{n}\left(u_{0 n}(x)\right) d x .
$$

Since $u_{n}$ and $\hat{u}_{n}$ are classical solutions it follows that (5.5) and (5.6) hold with $V, c, u$ and $\hat{u}$ replaced by $V_{n}, c_{n}, u_{n}$ and $\hat{u}_{n}$. Observe that (5.5) can be written as

$$
\begin{gathered}
\left.\int_{\Omega}\left\{q_{n}\left(u_{n}(x, t)\right)-\hat{u}_{n}(x) c_{n}\left(u_{n}(x, t)\right)\right\} d x\right|_{t_{1}} ^{t_{2}} \\
=-\int_{t_{1}}^{t_{2}} \int_{\Omega}\left|\nabla u_{n}(x, t)-\nabla \hat{u}_{n}(x)\right|^{2} d x d t
\end{gathered}
$$

for all $t_{1}, t_{2} \in[0, \infty)$ and that $V_{n}$ is convex. 
LEMMA 5.2. Let $V_{n}$ and $V$ be as above, and let $u_{n}$ denote the subsequence which converges to the weak solution $u$ of Problem I. Then $V_{n} \rightarrow V$ pointwise as $n \rightarrow \infty$.

PROOF. We set $v_{n}=c_{n}\left(u_{n}\right)$ and $v=c(u)$. As we observed above (2.21) holds for any $T>0$ and consequently

$$
v_{n}(\cdot, t) \rightarrow v(\cdot, t) \quad \text { in } L^{2}(\Omega)
$$

for any $t \geq 0$. Hence there exists for every fixed $t \geq 0$ a subsequence $v_{\mu}(\cdot, t)$ such that

$$
v_{\mu}(\cdot, t) \rightarrow v(\cdot, t) \quad \text { a.e. on } \Omega .
$$

By standard regularity theory we have that

$$
\hat{u}_{n} \rightarrow \hat{u} \quad \text { in } W^{1, p}(\Omega), p \geq 1 .
$$

Because of the $L^{\infty}$-bound on $u_{n}$, it follows from [7, Lemma 4] and (5.11) and (5.13), that

$$
q_{\mu}\left(u_{\mu}(\cdot, t)\right)-\hat{u}_{\mu} c_{\mu}\left(u_{\mu}(\cdot, t)\right) \rightarrow q(u(\cdot, t))-\hat{u} c(u(\cdot, t)) \quad \text { a.e. on } \Omega
$$

and thus, by Lebesgue's dominated convergence theorem, that

$$
V_{\mu}(t) \rightarrow V(t) \quad \text { as } \mu \rightarrow \infty .
$$

Since for any $t \geq 0$, any subsequence contains a further subsequence which satisfies (5.14), we have in fact that

$$
V_{n}(t) \rightarrow V(t) \quad \text { as } n \rightarrow \infty
$$

for all $t \geq 0$. This proves Lemma 5.2.

We continue with the proof of Lemma 5.1. By Lemma 5.2, the convexity of $V$ is a consequence of the convexity of $V_{n}$. To prove (5.7) we let $n$ tend to infinity in (5.10). Since

$$
\hat{u}_{n} \rightarrow \hat{u} \quad \text { in } H^{1}(\Omega)
$$

and

it follows that

$$
u_{n} \stackrel{*}{\rightarrow} u \quad \text { in } L^{\infty}\left(t_{1}, t_{2} ; H^{1}(\Omega)\right),
$$

$$
\nabla u_{n}-\nabla \hat{u}_{n}-\nabla u-\nabla \hat{u} \quad \text { in } L^{2}\left(\Omega \times\left(t_{1}, t_{2}\right)\right) .
$$

Thus the equality sign in (5.10) has to be replaced by an inequality sign in the limit. This completes the proof of Lemma 5.1.

PROOF OF THEOREM 1.2. From (5.10), (5.13) and the uniform bound on $\int_{\Omega}\left|\nabla u_{n}(x, t)\right|^{2} d x$ we derive that the sequence $V_{n}$ is bounded in $W^{1, \infty}\left(\mathbf{R}^{+}\right)$. By Lemma 5.1 we have that $V$ is convex and hence that $V^{\prime}$ is nondecreasing. Since $V$ is bounded we conclude that $V^{\prime}(t) \downarrow 0$ as $t \uparrow \infty$. But then Lemma 5.1 implies that

or

$$
0 \leq \int_{\Omega}|\nabla u(x, t)-\nabla \hat{u}(x)|^{2} d x \leq V^{\prime}(t) \downarrow 0,
$$

$$
\nabla u(\cdot, t) \rightarrow \nabla \hat{u} \quad \text { in } L^{2}(\Omega) \text { as } t \rightarrow \infty .
$$

Hence any sequence $t_{n} \uparrow \infty$ has a subsequence $t_{\mu} \uparrow \infty$ such that $u\left(\cdot, t_{\mu}\right)$ converges in $H^{1}(\Omega)$. Because of Proposition 2.7, the limit can only be $\hat{u}$. This completes the proof of Theorem 1.2. 
6. Asymptotic behavior for the nonautonomous problem. We now prove Theorem 1.3. Throughout this section we assume that $\mathrm{H1}-4$ hold for all $T>0$, that $f-g \in L^{1}\left(\partial \Omega \times \mathbf{R}^{+}\right)$, and that (1.10) holds.

PROOF OF THEOREM 1.3. Fix $\varepsilon>0$. Then there exists $T_{\varepsilon}>0$ such that

$$
0 \leq \int_{T_{\varepsilon}}^{\infty} \int_{\partial \Omega}\{f(x, t)-g(x)\} d x d t \leq \varepsilon
$$

Let $v=c(u)$ and let $u_{\varepsilon}$ be the weak bounded solution of

$$
\left\{\begin{array}{l}
(c(u))_{t}=\Delta u \quad \text { in } \Omega \times\left(T_{\varepsilon}, \infty\right), \\
\partial u / \partial \nu=g \quad \text { on } \partial \Omega \times\left(T_{\varepsilon}, \infty\right), \\
c\left(u\left(x, T_{\varepsilon}\right)\right)=v\left(x, T_{\varepsilon}\right), \quad x \in \Omega .
\end{array}\right.
$$

By Theorem 1.2, this solution exists and

$$
c\left(u_{\varepsilon}(\cdot, t)\right) \rightarrow c\left(\hat{u}_{\varepsilon}\right) \quad \text { in } L^{1}(\Omega) \quad \text { as } t \rightarrow \infty,
$$

where $\hat{u}_{\varepsilon}$ is the solution of Problem II which satisfies

$$
\int_{\Omega} c\left(\hat{u}_{\varepsilon}(x)\right)=\int_{\Omega} v_{0}(x) d x+\int_{0}^{T_{\varepsilon}} \int_{\partial \Omega} f(x, t) d x d t .
$$

Note that the difference between $\hat{u}$ and $\hat{u}_{\varepsilon}$ is a constant depending only on $\varepsilon$ and that

$$
b(\varepsilon) \stackrel{\text { def }}{=}\left\|c\left(\hat{u}_{\varepsilon}\right)-c(\hat{u})\right\|_{L^{1}(\Omega)} \rightarrow 0 \quad \text { as } \varepsilon \rightarrow 0 .
$$

By the comparison principle (Theorem 4.3),

$$
c\left(u_{\varepsilon}\right) \leq c(u) \quad \text { on } \Omega \times\left(T_{\varepsilon}, \infty\right),
$$

and (6.1) implies, with Proposition 2.7,

$$
0 \leq \int_{\Omega}\left\{c(u(x, t))-c\left(u_{\varepsilon}(x, t)\right)\right\} d x \leq \varepsilon
$$

for all $t \geq T_{\varepsilon}$. Thus, using the triangle inequality

$$
\begin{aligned}
\|c(u(\cdot, t))-c(\hat{u})\|_{L^{1}(\Omega)} & \leq\left\|c(u(\cdot, t))-c\left(u_{\varepsilon}(\cdot, t)\right)\right\|_{L^{1}(\Omega)} \\
& +\left\|c\left(u_{\varepsilon}(\cdot, t)\right)-c\left(\hat{u}_{\varepsilon}\right)\right\|_{L^{1}(\Omega)}+\left\|c\left(\hat{u}_{\varepsilon}\right)-c(\hat{u})\right\|_{L^{1}(\Omega)} .
\end{aligned}
$$

Hence, by (6.2), (6.5) and (6.6),

$$
\limsup _{t \rightarrow \infty}\|c(u(\cdot, t))-c(\hat{u})\|_{L^{1}(\Omega)} \leq b(\varepsilon),
$$

for every $\varepsilon>0$ and Theorem 1.3 follows from (6.4) and (6.8). Note that (1.12) follows from Theorem 1.3, Theorem 2.5 and the Arzéla-Ascoli Theorem.

ACKnowledgment. I would like to thank M. Bertsch, F. Beukers, L. A. Peletier and J. F. Rodrigues for a number of fruitful discussions. 


\section{REFERENCES}

1. H. W. Alt and S. Luckhaus, Quasilinear elliptic-parabolic differential equations, Math. Z. 183 (1983), 311-341.

2. M. Bertsch and J. Hulshof, Regularity results for an elliptic-parabolic free boundary problem, Trans. Amer. Math. Soc. 297 (1986), 337-350.

3. E. DiBenedetto and R. Gariepy, Local behavior of solutions of an elliptic-parabolic equation, preprint.

4. C. J. Van Duyn, Nonstationary filtration in partially saturated media: continuity of the free boundary, Arch. Rational Mech. Anal. 79 (1982), 261-265.

5. C. J. Van Duyn and L. A. Peletier, Nonstationary filtration in partially saturated porous media, Arch. Rational Mech. Anal. 78 (1982), 173-198.

6. J. Hulshof, An elliptic-parabolic problem: continuity of the interface, Rep. No. 10, Math. Inst. of Leiden University, 1985.

7. J. Hulshof and L. A. Peletier, An elliptic-parabolic free boundary problem, Rep. No. 14, Math. Inst. of Leiden University, 1984.

8. D. Kröner and J. F. Rodrigues, Global behavior for bounded solutions of a porous media equation of elliptic-parabolic type, Report, Bonn Univ., 1983.

9. O. A. Ladyženskaja, V. A. Solonnikov and N. N. Ural'ceva, Linear and quasilinear equations of parabolic type, Transl. Math. Monographs, vol. 23, Amer. Math. Soc., Providence, R.I., 1968.

Mathematical Institute, University of Leiden, Leiden, The Netherlands 